\title{
Improving 3D scan matching time of the coarse binary cubes method with fast spatial subsampling
}

\author{
Jesús Morales, Jorge L. Martínez, Anthony Mandow, Antonio. J. Reina, Javier Serón \\ and A. García-Cerezo \\ Universidad de Málaga - Andalucía Tech \\ Departamento de Ingeniería de Sistemas y Automática, 29071 Málaga, Spain. \\ Email: jesus.morales[@]uma.es
}

\begin{abstract}
Exploiting the huge amount of real time range data provided by new multi-beam three-dimensional (3D) laser scanners is challenging for vehicle and mobile robot applications. The Coarse Binary Cube (CBC) method was proposed to achieve fast and accurate scene registration by maximizing the number of coincident cubes between a pair of scans. The aim of this paper is speeding up CBC with a fast spatial subsampling strategy for raw point clouds that employs the same type of efficient data structures as CBC. Experimental results have been obtained with the Velodyne HDL-32E sensor mounted on the Quadriga mobile robot on irregular terrain. The influence of the subsampling rate has been analyzed. Preliminary results show a relevant gain in computation time without losing matching accuracy.
\end{abstract}

Keywords: mobile robots, optical scanners, sampling methods, velodyne, irregular terrain, 3D scan matching..

This document is a self-archiving copy of the accepted version of the paper.

Please find the final published version in IEEEXplore: http://dx.doi.org/10.1109/IECON.2013.6699804

\section{Citation Information:}

Morales, J.; Martinez, J.L.; Mandow, A.; Reina, A.J.; Seron, J.; Garcia-Cerezo, A.,

"Improving 3D scan matching time of the coarse binary cubes method with fast spatial subsampling," 39th Annual Conference of the IEEE Industrial Electronics Society, pp. 4168-4173, 2013

doi:10.1109/IECON.2013.6699804

@INPROCEEDINGS $\{$ Morales: IECON2013,

author $=\{$ J. Morales and J. L. Mart $\backslash\{\{i\} n e z$ and A. Mandow and A. J. Reina and J. Ser $\backslash\{$ ' o $\}$ n and A. Garc $\backslash\{i\} a-C e r e z o\}$, title $=\{$ Improving $\{3 \mathrm{D}\}$ scan matching time of the coarse binary cubes method with fast spatial subsampling $\}$, booktitle $=\{39$ th Annual Conference of the IEEE Industrial Electronics Society $\}$, year $=\{2013\}$

address $=\{$ Vienna, Austria $\}$ pages $=\{4168-4173\}$,

\}

C 2013 IEEE. Personal use of this material is permitted. Permission from IEEE must be obtained for all other uses, in any current or future media, including reprinting/republishing this material for advertising or promotional purposes, creating new collective works, for resale or redistribution to servers or lists, or reuse of any copyrighted component of this work in other works. 


\title{
Improving 3D Scan Matching Time of the Coarse Binary Cubes Method with Fast Spatial Subsampling
}

\author{
Jesús Morales, Jorge L. Martínez, Anthony Mandow, Antonio J. Reina, Javier Serón and Alfonso García-Cerezo \\ Dpto. Ingeniería de Sistemas y Automática, Universidad de Málaga, 29071 Málaga, Spain \\ Email: jesus.morales@uma.es, Tel: (+34) 951952323.
}

\begin{abstract}
Exploiting the huge amount of real time range data provided by new multi-beam three-dimensional (3D) laser scanners is challenging for vehicle and mobile robot applications. The Coarse Binary Cube (CBC) method was proposed to achieve fast and accurate scene registration by maximizing the number of coincident cubes between a pair of scans. The aim of this paper is speeding up CBC with a fast spatial subsampling strategy for raw point clouds that employs the same type of efficient data structures as CBC. Experimental results have been obtained with the Velodyne HDL-32E sensor mounted on the Quadriga mobile robot on irregular terrain. The influence of the subsampling rate has been analyzed. Preliminary results show a relevant gain in computation time without losing matching accuracy.
\end{abstract}

\section{INTRODUCTION}

Recently introduced multi-beam three-dimensional (3D) laser scanners provide a huge amount of range data in real time [1] [2] [3]. These point clouds can be employed for mobile robot localization [4] [5] and for tracking mobile objects from road vehicles [6] [7] [8].

Some 3D scene registration methods [9] have been adapted from well-known object registration techniques such as Iterative Closest Points (ICP) [10] [11]. To improve robustness of ICP against initial misalignments due to odometric estimations, a pre-processing step can perform either scan segmentation to extract objects [12] or coarse octree alignment in an exhaustive discrete search [13].

Specialized methods for pairwise scene alignment like Normal Distribution Transform (NDT) [14] and the Coarse Binary Cubes technique (CBC) [15] improve performance by avoiding computation of nearest neighbor points. Furthermore, CBC explicitly takes into account that all scan directions and depths can contain relevant data. Precisely, the point clouds from commercial multi-beam scanners provide sparse data regions due to limited vertical resolution [16]. Moreover, spherical scanning mechanisms yield varying densities depending on the distance to the sensor [17] [18].

CBC maximizes the number of coincident cubes between a pair of raw scans [15]. The implementation of this method with efficient one-dimensional data structures allows achieving better computation times than ICP and NDT for similar registration accuracy [19]. Recently, we addressed parallelization of the $\mathrm{CBC}$ optimization to profit from widespread multi-core and multi-thread processors [20], where the maximum gain is limited by the number of processor cores. Nevertheless, further progress can be crucial to meet the demanding requirements of vehicle-based applications. From a software perspective, additional computation time improvement can be gained by applying effective subsampling procedures to raw 3D scans [21].

The major contribution of this paper is introducing spatial subsampling to speed up CBC pairwise scene alignment without degrading matching accuracy. In particular, a fast subsampling strategy is proposed which employs the same type of efficient data structures as CBC. Experimental results have been obtained with the Velodyne HDL-32E sensor mounted on the mobile robot Quadriga. The influence of the subsampling rate on accuracy and computation time has been analyzed for scans on irregular terrain.

The paper is organized as follows. Next section briefly reviews the CBC method. Section III proposes the subsampling strategy to be applied to $\mathrm{CBC}$. Experimental results are presented in section IV. Finally, the last section is devoted to conclusions and future work.

\section{THE CBC REgISTRATION TECHNIQUE}

CBC looks for the spatial transformation $\mathbf{T}=\left[x_{0}, y_{0}, z_{0}, \alpha, \beta, \gamma\right]$ that maximizes the number $J$ of coincident 3D binary occupancy cubes between the first range image and the projection of the second range image according to $\mathbf{T}$ in a 3D spatial grid (see Fig. 1). This grid is defined in the frame of the first range image with regularly sized cubes of edge length $E$ [19].

Cost function $J(\mathbf{T})$ is computed with the following data structures:

- $V$ is a binary vector whose elements correspond to the cubes contained by the axis-aligned minimum bounding box for the actual readings of the first range image. Each cube has a unique integer index $I$ in $V$.

- $L$ is an unsorted integer list that contains the same information as $V$ in a non-sparse way. The length $\ell$ of $L$ corresponds to the number of cubes set to one, which is always less or equal than the number $n$ of points from the first scan. Note that the relation $\ell \ll n$ holds with coarse cubes.

In CBC, the first scan is processed once to build $V$ and $L$ (see Fig. 2). Initially, $V$ is created as a zero vector of length $v$, and $L$ is empty. Then, the $I$ index is computed for each point in the first range image. If $V(I)=0$, then $V(I)$ is set to one, and $I$ is inserted into $L$.

Let $x_{\max }, x_{\min }, y_{\max }, y_{\min }, z_{\max }, z_{\min }$ be the Cartesian coordinates of the minimum bounding box for the points of the first point cloud. Then, the length $v$ of $V$ is given by:

$$
v=I_{x \max } I_{y \max } I_{z \max },
$$




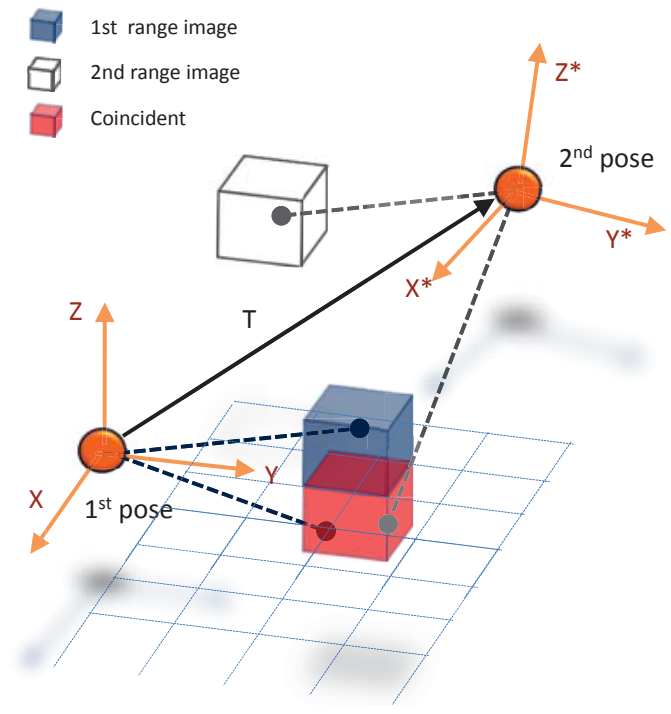

Figure 1. The CBC principle [19].

where

$$
\begin{aligned}
& I_{x \max }=\operatorname{round}\left(\frac{x_{\max }-x_{\text {min }}}{E}\right)+1, \\
& I_{y \max }=\operatorname{round}\left(\frac{y_{\max }-y_{\min }}{E}\right)+1, \\
& I_{z \max }=\operatorname{round}\left(\frac{z_{\max }-z_{\min }}{E}\right)+1 .
\end{aligned}
$$

The index $I$ of a Cartesian scan point $x, y, z$ from the first scan is:

$$
I=I_{x}+I_{y} I_{x \max }+I_{z} I_{x \max } I_{y \max },
$$

where $I_{x}, I_{y}, I_{z}$ are the following integer grid coordinates:

$$
\begin{aligned}
& I_{x}=\operatorname{round}\left(\frac{x-x_{\text {min }}}{E}\right), \\
& I_{y}=\operatorname{round}\left(\frac{y-y_{\text {min }}}{E}\right), \\
& I_{z}=\operatorname{round}\left(\frac{z-z_{\text {min }}}{E}\right) .
\end{aligned}
$$

The objective function $J$ has to be evaluated for each prospective solution $\mathbf{T}$ in the optimization process. Prior to each evaluation, $J$ is initialized to zero. Next, for each point of the second range image, its projection is obtained according to $\mathbf{T}$. Then, its $I$ index is computed if the projected Cartesian coordinates fall within the minimum boundary box for the first range image. In this case, if $V(I)=1$, then $V(I)$ is set to zero and $J$ is incremented by one. Once $J$ has been obtained for a prospective solution, $V$ can be recovered from $L$ for a new evaluation.

To search for a $\mathbf{T}$ that maximizes $J(\mathbf{T})$, CBC employs a globalized variation of the Nelder-Mead method [22] that pursues escaping from local optima [19].

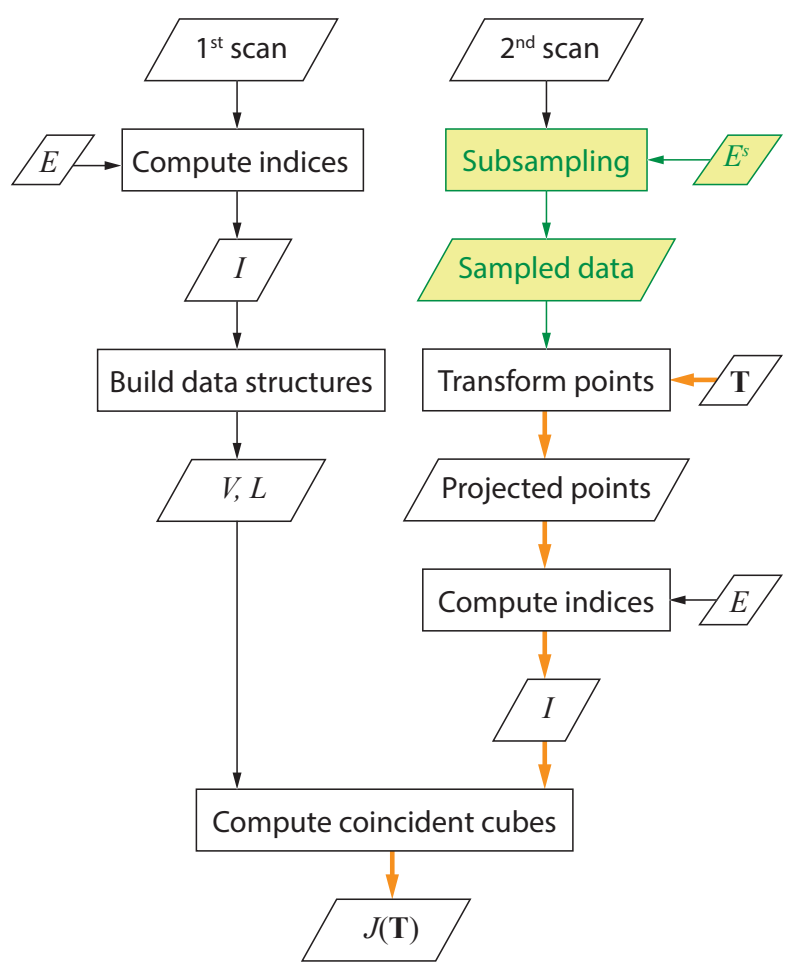

Figure 2. Flowchart for evaluating the $\mathrm{CBC}$ cost function. In shaded green colour: new subsampling stage. Thick orange arrows: computations for a single transformation $\mathbf{T}$.

\section{SUBSAMPLING STRATEGY}

Evaluation of numerous prospective solutions in the NelderMead search is the bottleneck of the CBC method. Furthermore, each evaluation of $\mathbf{T}$ requires projecting Cartesian coordinates as well as calculating $I$ indices for every point of the second scan. This is highlighted in Fig. 2 with thick orange arrows. Therefore, $\mathrm{CBC}$ can be sped up if a reduced but representative set of points is chosen from the second scan. This new subsampling step is shown in Fig. 2 with shaded green color.

There are many subsampling procedures that can be applied to raw 3D scans [18] [21]. Among them, choosing octree cube centers [9] is closely related with the uniform spatial representation implicitly used by $\mathrm{CBC}$. This simple method starts with a cube containing the whole 3D scan. Then, the cube is divided recursively into eight octants. Empty octants are pruned and the subdivision continues until a minimal octant size $E^{s}$ is reached. Finally, the centers of the occupied cubes are collected resulting in a subsampled set of $m$ points.

Alternatively, we propose obtaining a subsampled set identical to octree centers by profiting from the same type of data structures employed by CBC for the first scan: $V$ and $L$ (see Section II). The equivalent subsampling structures $V^{s}$ and $L^{s}$ are computed in the Cartesian frame of the second scan and with cube edges of length $E^{s}$. Thus, the use of complex data structures such as octrees is avoided. 
The coordinates $x^{s}, y^{s}, z^{s}$ of the center of the cubes set to one are obtained from the list $L^{s}$ of indices $I^{s}$ as the reciprocals of (5) to (8):

$$
\begin{aligned}
& x^{s}=I_{x}^{s} E^{s}+x_{\min }^{s}, \\
& y^{s}=I_{y}^{s} E^{s}+y_{\min }^{s}, \\
& z^{s}=I_{z}^{s} E^{s}+z_{\text {min }}^{s}
\end{aligned}
$$

where

$$
\begin{aligned}
& I_{x}^{s}=\operatorname{remainder}\left(\frac{I^{s}}{I_{x \max }^{s}}\right), \\
& I_{y}^{s}=\operatorname{remainder}\left(\frac{\left(I^{s}-I_{x}^{s}\right) / I_{x \max }^{s}}{I_{y \max }^{s}}\right), \\
& I_{z}^{s}=\frac{I^{s}-I_{x}^{s}-I_{y}^{s} I_{x \max }^{s}}{I_{x \max }^{s} I_{y \max }^{s}} .
\end{aligned}
$$

\section{EXPERIMENTS}

\section{A. Experimental Setup}

The method has been tested on scans acquired with the Velodyne HDL-32E sensor. This scanner employs and array of 32 lasers/detectors pairs located on a rotating head that spins at $10 \mathrm{~Hz}$. It provides a measurement range of $1 \mathrm{~m}$ to $100 \mathrm{~m}$ with an accuracy of $\pm 2 \mathrm{~cm}$ for distances below $70 \mathrm{~m}$. The vertical field of view is $41.34^{\circ}$ with a fixed resolution of $1.33^{\circ}$, and the horizontal field of view is $360^{\circ}$ with a minimum resolution of $0.16^{\circ}$.

The laser rangefinder is mounted $0.82 \mathrm{~m}$ above the ground on the 4-wheel skid-steer mobile robot Quadriga [23] (see Fig. 3). With the minimum horizontal resolution, the resulting range images have a maximum of 72000 points, with a scanning time of $0.1 \mathrm{~s}$. Nevertheless, the number of points of a scan is smaller because sky points are discarded. Moreover, given the maximum sensor range, measurements can spread over a large area of $\pi$ hectares.

A continuous sequence of point clouds with $10 \mathrm{~Hz}$ scanning frequency has been obtained during the nonstop forward motion of Quadriga on irregular terrain (see Fig. 4). The first scan of this sequence is presented in Fig. 5. The characteristic scanning pattern and the uneven point distribution of the HDL$32 \mathrm{E}$ sensor are noticeable in this figure.

A second scan has been selected after the robot advanced a few meters from the initial pose. A ground truth spatial transformation with respect to the first scan has been adjusted manually by aligning salient features, resulting in $\mathbf{T}_{\mathrm{gt}}=$ $\left[4.75 \mathrm{~m}, 2.92 \mathrm{~m}, 0.29 \mathrm{~m}, 168.53^{\circ}, 2.52^{\circ}, 3.70^{\circ}\right]$ with the RollPitch-Yaw convention, and with axis $\mathrm{Y}$ and $\mathrm{Z}$ pointing forward and upward, respectively.

\section{B. Registration results}

Registration of both scans has been evaluated in a PC with an Intel Core i7 2720QM (2.20 GHz, 6 MByte cache, 8 GByte RAM) processor under the Linux operating system.

The CBC search has been carried out with 1000 evaluations of the cost function $J$. The size of the cube edge is $E=$ $0.9 \mathrm{~m}$. Initial estimations for each match have been obtained

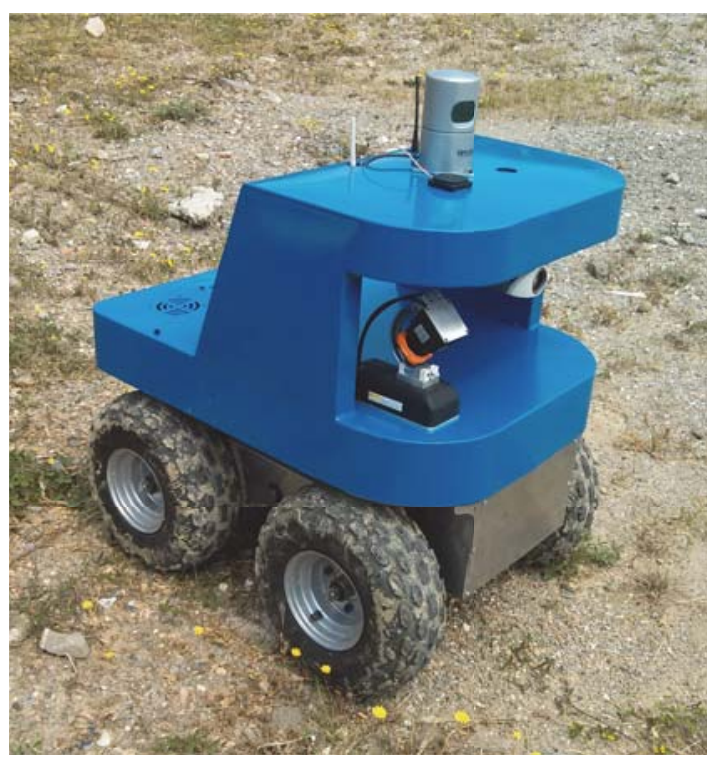

Figure 3. The Quadriga mobile robot with the HDL-32E sensor mounted on top.

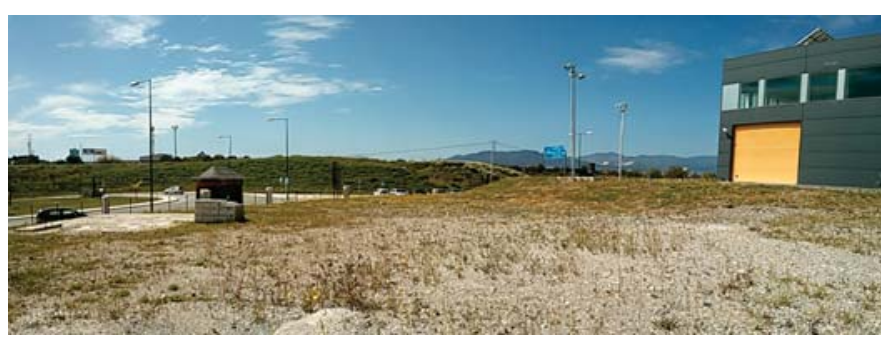

Figure 4. Photograph of the outdoor environment.

by adding large random errors within $\pm 1 \mathrm{~m}$ for translations and $\pm 8^{\circ}$ for rotations to the ground truth. A CBC alignment obtained for the experimental data with no subsampling is shown in Fig. 6.

Fig. 7 shows the resulting subsampled points with $E^{s}=$ $0.9 \mathrm{~m}$. Subsampling times range from $4 \mathrm{~ms}$ to $9 \mathrm{~ms}$ for $E^{s}=0.9 \mathrm{~m}$ and $E^{s}=0.2 \mathrm{~m}$, respectively. This is due to initialization of $V^{s}$, which is needed once for the whole registration process. For comparison purposes, the same sets of subsampled points have been computed as octree cube centers [9] by using the Point Cloud Library [24]. In this case, subsampling times vary from $7 \mathrm{~ms}$ to $10 \mathrm{~ms}$, which are longer than those achieved by the proposed solution.

For registration accuracy evaluation, the following errors in distance $D_{s}$ and in orientation $D_{a}$ are defined:

$$
\begin{aligned}
& D_{s}=\sqrt{\Delta x^{2}+\Delta y^{2}+\Delta z^{2}}, \\
& D_{a}=\sqrt{\Delta \alpha^{2}+\Delta \beta^{2}+\Delta \gamma^{2}},
\end{aligned}
$$

where $\Delta x, \Delta y, \Delta z$ and $\Delta \alpha, \Delta \beta, \Delta \gamma$ are the differences with respect to the ground truth.

Table I shows the influence of $E^{s}$ on accuracy and computation time. The ratio of subsampled points $r$ is also given. Each row offers the average values of 40 registrations with 


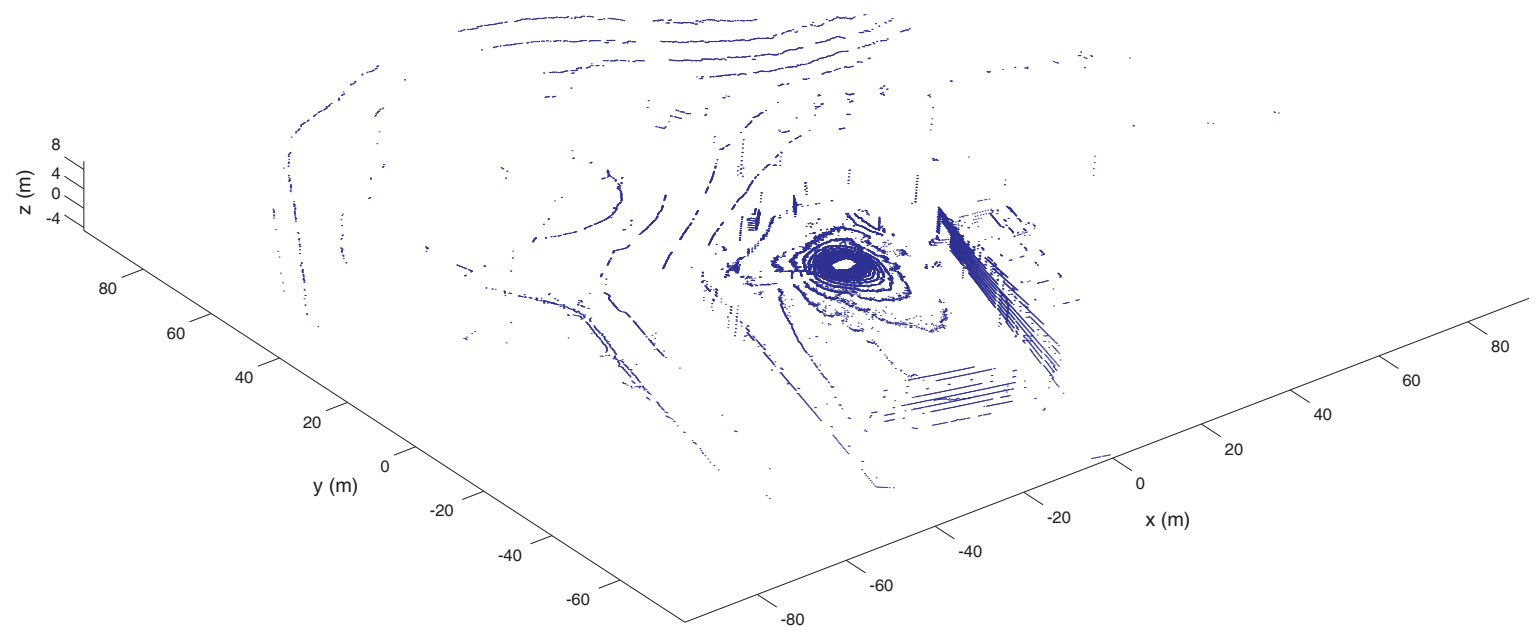

Figure 5. Point cloud for the first scan.

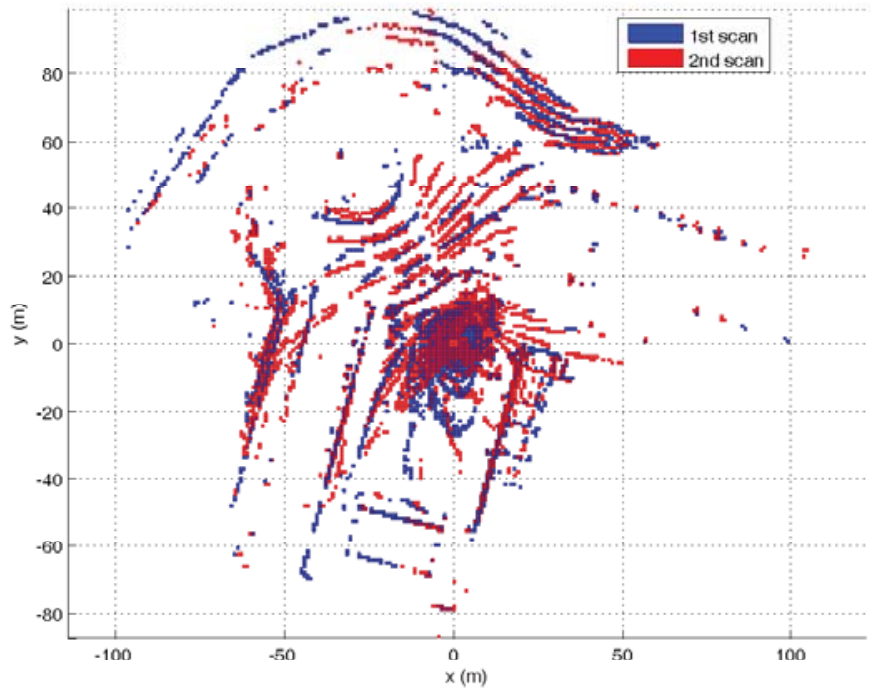

Figure 6. Top view of an alignment with $\mathrm{CBC}$ and no subsampling.

different random initial estimations. All in all, the table offers results from 640 different matches performed by CBC.

Computation times from the table have been summarized in Fig. 8. It can be seen that registration time reduces almost linearly with $r$. Besides, the gain in registration time of $\mathrm{CBC}$ with respect to the non-subsampled case also varies linearly with $E^{s}$.

As for the relation of subsampling parameter $E^{s}$ with CBC parameter $E$, it is noticeable that around $E^{s}=0.225 \mathrm{~m}$, i.e., a cube edge ratio $E / E^{s}=4$, the number of coincident cubes is almost the same as without subsampling. This means that the reduced set is very representative of the whole scan. Moreover, the $D_{s}$ and $D_{a}$ values in the table show that registration accuracy only degrades when $E^{s}$ approaches $E$. Therefore, a relation $E / E^{s} \approx 3$ is an acceptable compromise between accuracy and computation time.

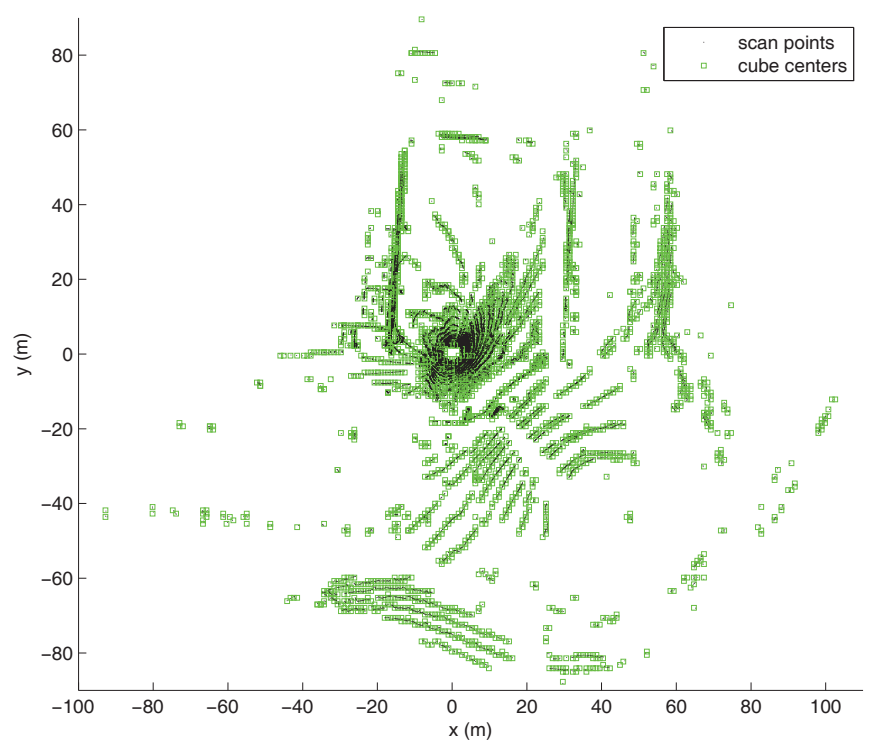

Figure 7. Top view of the subsampled points for the second scan with $E^{s}=$ $0.9 \mathrm{~m}$.

\section{CONClusions}

In this paper, the Coarse Binary Cubes (CBC) pairwise scene alignment is sped up by introducing a subsampling step which selects a reduced and representative set of points from the point cloud to be projected. For this purpose, a new spatial subsampling procedure has been defined that employs the same type of efficient data structures as CBC.

Experimental results have been obtained with the Velodyne HDL-32E sensor mounted on the Quadriga mobile robot on irregular terrain. The proposed subsampling method improves the times given by octree computation. The influence of the subsampling rate on $\mathrm{CBC}$ has been analyzed. Preliminary results show that subsampling can achieve a relevant gain in 
registration time without degrading matching accuracy.

Future work includes combining subsampling with parallel execution of $\mathrm{CBC}$ via multi-core processors [20] to achieve matching times less than scan acquisition times.

Table I

Average REgistration RESUlts With CBC $(E=0.9 \mathrm{~m})$.

\begin{tabular}{|c||c|c|c|c|c|c|c|}
\hline $\begin{array}{c}E^{s} \\
(\mathrm{~m})\end{array}$ & $m$ & $\begin{array}{c}r \\
(\%)\end{array}$ & $J\left(\mathbf{T}_{\mathbf{g t}}\right)$ & $J(\mathbf{T})$ & $\begin{array}{c}D_{s} \\
(\mathrm{~m})\end{array}$ & $\begin{array}{c}D_{a} \\
\left({ }^{o}\right)\end{array}$ & $\begin{array}{c}\text { Time } \\
(\mathrm{s})\end{array}$ \\
\hline \hline- & 65843 & 100.0 & 1583 & 1613 & 0.072 & 0.230 & 2.127 \\
\hline 0.200 & 16960 & 25.8 & 1566 & 1615 & 0.091 & 0.069 & 0.585 \\
\hline 0.212 & 16161 & 24.5 & 1578 & 1595 & 0.074 & 0.114 & 0.558 \\
\hline 0.225 & 15462 & 23.5 & 1566 & 1598 & 0.062 & 0.080 & 0.543 \\
\hline 0.240 & 14527 & 22.1 & 1558 & 1570 & 0.112 & 0.139 & 0.493 \\
\hline 0.257 & 13676 & 20.8 & 1555 & 1588 & 0.069 & 0.125 & 0.460 \\
\hline 0.277 & 12819 & 19.5 & 1548 & 1582 & 0.143 & 0.068 & 0.441 \\
\hline 0.300 & 11878 & 18.0 & 1551 & 1571 & 0.066 & 0.175 & 0.405 \\
\hline 0.327 & 11015 & 16.7 & 1553 & 1583 & 0.062 & 0.093 & 0.380 \\
\hline 0.360 & 10117 & 15.4 & 1534 & 1554 & 0.105 & 0.189 & 0.352 \\
\hline 0.400 & 9079 & 13.8 & 1497 & 1525 & 0.096 & 0.125 & 0.320 \\
\hline 0.450 & 8156 & 12.4 & 1506 & 1526 & 0.059 & 0.093 & 0.288 \\
\hline 0.514 & 7038 & 10.7 & 1456 & 1463 & 0.052 & 0.141 & 0.257 \\
\hline 0.600 & 6059 & 9.2 & 1399 & 1421 & 0.064 & 0.252 & 0.227 \\
\hline 0.720 & 4935 & 7.5 & 1334 & 1358 & 0.149 & 0.139 & 0.192 \\
\hline 0.900 & 3873 & 5.9 & 1238 & 1302 & 0.243 & 0.308 & 0.149 \\
\hline
\end{tabular}

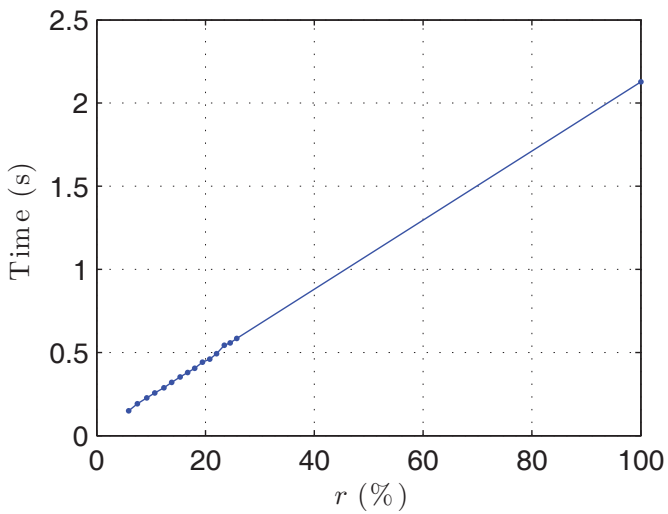

(a)

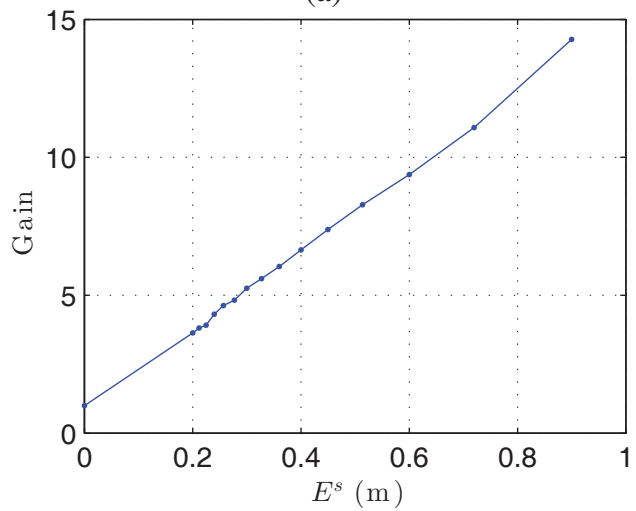

(b)

Figure 8. Computation time results: registration time versus $r$ (a), and computation time gain with respect to $E^{s}$ (b)

\section{ACKNOWLEDGMENTS}

This work was partially supported by the Spanish CICYT project DPI 2011-22443 and the Andalusian project PE-2010 TEP-6101.

\section{REFERENCES}

[1] C. Glennie and D. D. Lichti, "Static calibration and analysis of the Velodyne HDL-64E S2 for high accuracy mobile scanning," Remote Sensing, vol. 2, no. 6, pp. 1610-1624, 2010.

[2] — " "Temporal stability of the Velodyne HDL-64E S2 scanner for high accuracy scanning applications," Remote Sensing, vol. 3, no. 3, pp. 539-553, 2011.

[3] F. Marino, P. De Ruvo, G. De Ruvo, M. Nitti, and E. Stella, "HiPER 3-D: An omnidirectional sensor for high precision environmental 3-D reconstruction," IEEE Transactions on Industrial Electronics, vol. 59, no. 1, pp. 579-591, 2012.

[4] N. Muhammad and S. Lacroix, "Loop closure detection using smallsized signatures from 3D LIDAR data," in Proc. IEEE International Symposium on Safety, Security, and Rescue Robotics, Kyoto, Japan, 2011, pp. 333-338.

[5] F. Moosmann and C. Stiller, "Velodyne SLAM," in Proc. IEEE Intelligent Vehicles Symposium, Baden-Baden, Germany, 2011, pp. 393-398.

[6] A. Petrovskaya and S. Thrun, "Model based vehicle detection and tracking for autonomous urban driving," Autonomous Robots, vol. 26, no. 2-3, pp. 123-139, 2009.

[7] N. Wojke and M. Häselich, "Moving vehicle detection and tracking in unstructured environments," in Proc. IEEE International Conference on Robotics and Automation, Saint Paul, USA, 2012, pp. 3082-3087.

[8] A. Azim and O. Aycard, "Detection, classification and tracking of moving objects in a 3D environment," in Proc. IEEE Intelligent Vehicles Symposium, Alcalá de Henares, Spain, 2012, pp. 802-807.

[9] A. Nüchter, "3D range image registration," in 3D Robotic Mapping: The Simultaneous Localization and Mapping Problem with Six Degrees of Freedom. Springer, 2009, pp. 35-75.

[10] P. J. Besl and N. D. McKay, "A method for registration of 3-D shapes," IEEE Transactions on Pattern Analysis and Machine Intelligence, vol. 14, no. 2, pp. 239-256, 1992.

[11] Z. Zhang, "Iterative point matching for registration of free-form curves and surfaces," International Journal on Computer Vision, vol. 13, no. 2 , pp. 119-152, 1994.

[12] B. Douillard, A. Quadros, P. Morton, J. P. Underwood, M. De Deuge, S. Hugosson, M. Hallström, and T. Bailey, "Scan segments matching for pairwise 3D alignment," in Proc. IEEE International Conference on Robotics and Automation, Saint Paul, USA, 2012, pp. 3033-3040.

[13] A. Nüchter, K. Lingemann, J. Hertzberg, and H. Surmann, "6D SLAM 3D mapping outdoor environments," Journal of Field Robotics, vol. 24, no. 8-9, pp. 699-722, 2007.

[14] M. Magnusson, A. Lilienthal, and T. Duckett, "Scan registration for autonomous mining vehicles using 3D-NDT," Journal of Field Robotics, vol. 24, no. 10, pp. 803-827, 2007.

[15] J. L. Martínez, A. Mandow, A. Reina, and J. Morales, "Outdoor scene registration from 3D laser range data with coarse binary cubes," in Proc. Annual Conference of the IEEE Industrial Electronics Society, Porto, Portugal, 2009, pp. 2308-2313.

[16] B. Douillard, J. Underwood, N. Kuntz, V. Vlaskine, A. Quadros, P. Morton, and A. Frenkel, "On the segmentation of 3D LIDAR point clouds,' in Proc. IEEE International Conference on Robotics and Automation, Shanghai, China, 2011, pp. 2798-2805.

[17] O. Wulf and B. Wagner, "Fast 3D scanning methods for laser measurement systems," in Proc. International Conference on Control Systems and Computer Science, Bucharest, Romania, 2003, pp. 312-317.

[18] A. Mandow, J. L. Martínez, A. J. Reina, and J. Morales, "Fast rangeindependent spherical subsampling of 3D laser scanner points and data reduction performance evaluation for scene registration," Pattern Recognition Letters, vol. 31, pp. 1239-1250, 2010.

[19] J. L. Martínez, A. J. Reina, A. Mandow, and J. Morales, "3D registration of laser range scenes by coincidence of coarse binary cubes," Machine Vision and Applications, vol. 23, pp. 857 -867, 2012. 
[20] J. L. Martínez, A. J. Reina, J. Morales, A. Mandow, and A. GarcíaCerezo, "Using multicore processors to parallelize 3D point cloud registration with the coarse binary cubes method," in Proc. IEEE International Conference on Mechatronics, Vicenza, Italy, 2013, pp. 335-340

[21] S. Rusinkiewicz and M. Levoy, "Efficient variants of the ICP algorithm,' in Proc. International Conference on 3D Digital Imaging and Modeling, Quebec, Canada, 2001, pp. 145-152.
[22] J. A. Nelder and R. Mead, "A simplex method for function minimization," The Computer Journal, vol. 7, no. 4, pp. 308-313, 1965.

[23] J. Morales, J. L. Martínez, A. Mandow, A. Pequeño-Boter, and A. García-Cerezo, "Simplified power consumption modeling and identification for wheeled skid-steer robotic vehicles on hard horizontal ground," in Proc. IEEE/RSJ International Conference on Intelligent Robots and Systems, Taipei, Taiwan, 2010, pp. 4769-4774.

[24] R. B. Rusu and S. Cousins, "3D is here: Point Cloud Library (PCL)," in Proc. IEEE International Conference on Robotics and Automation, Shanghai, China, 2011, pp. 1-4. 